\title{
Insights into Caco-2 cell culture structure using coherent anti-Stokes Raman scattering (CARS) microscopy
}

\section{Saarinen, Jukka}

2017-05-15

Saarinen , J , Sõzeri , E , Fraser-Miller , S , Peltonen , L , A. Santos , H , Isomäki , A \& Strachan , C J 2017 , ' Insights into Caco-2 cell culture structure using coherent anti-Stokes Raman scattering (CARS) microscopy ' , International Journal of Pharmaceutics , vol. 523 , pÿno. 1 , pp. 270280 . https://doi.org/10.1016/j.ijpharm.2017.03.015

http://hdl.handle.net/10138/235583

https://doi.org/10.1016/j.jpharm.2017.03.015

unspecified

publishedVersion

Downloaded from Helda, University of Helsinki institutional repository.

This is an electronic reprint of the original article.

This reprint may differ from the original in pagination and typographic detail.

Please cite the original version. 


\title{
Insights into Caco-2 cell culture structure using coherent anti-Stokes Raman scattering (CARS) microscopy
}

\author{
Jukka Saarinen $^{\mathrm{a}, *}$, Erkan Sözeri ${ }^{\mathrm{a}}$, Sara J. Fraser-Miller ${ }^{\mathrm{a}}$, Leena Peltonen ${ }^{\mathrm{a}}$, \\ Hélder A. Santos ${ }^{\mathrm{a}}$, Antti Isomäki ${ }^{\mathrm{b}}$, Clare J. Strachan ${ }^{\mathrm{a}}$ \\ a Division of Pharmaceutical Chemistry and Technology, Faculty of Pharmacy, FI-00014 University of Helsinki, Finland \\ ${ }^{\mathrm{b}}$ Biomedicum Imaging Unit, Department of Anatomy, Faculty of Medicine, FI-00014 University of Helsinki, Finland
}

\section{A R T I C L E I N F O}

\section{Article history:}

Received 25 January 2017

Received in revised form 7 March 2017

Accepted 8 March 2017

Available online 12 March 2017

\section{Keywords:}

Caco-2 cells

Live cell imaging

CARS microscopy

Lipid droplets

Non-linear imaging

Raman spectroscopy

\begin{abstract}
A B S T R A C T
We have used coherent anti-Stokes Raman scattering (CARS) microscopy as a novel and rapid, label-free and non-destructive imaging method to gain structural insights into live intestinal epithelial cell cultures used for drug permeability testing. Specifically we have imaged live Caco-2 cells in (bio)pharmaceutically relevant conditions grown on membrane inserts. Imaging conditions were optimized, including evaluation of suitable membrane materials and media solutions, as well as tolerable laser powers for non-destructive imaging of the live cells. Lipid structures, in particular lipid droplets, were imaged within the cells on the insert membranes. The size of the individual lipid droplets increased substantially over the 21-day culturing period up to approximately $10 \%$ of the volume of the cross section of individual cells. Variation in lipid content has important implications for intestinal drug permeation testing during drug development but has received limited attention to date due to a lack of suitable analytical techniques. CARS microscopy was shown to be well suited for such analysis with the potential for in situ imaging of the same individual cell-cultures that are used for permeation studies. Overall, the method may be used to provide important information about cell monolayer structure to better understand drug permeation results.
\end{abstract}

(C) 2017 Elsevier B.V. All rights reserved.

\section{Introduction}

Caco-2 cells have been widely used as in vitro cell models to study intestinal drug permeation in the early stages of drug development (Shah et al., 2006). According to the Web of Science database, about 13000 publications involving Caco-2 cells have been published from 2000 to 2015, with the annual number of publications increasing over this period (Fig. S1.). In the field of

Abbreviations: CARS, coherent anti-Stokes Raman scattering; DMEM, Dulbecco's Modified Eagle's Medium; FBS, fetal bovine serum; NEAA, non-essential amino acids; EDTA, ethylenediaminetetraacetic acid disodium salt dihydrate; HEPES, 2-[4(2-hydroxyethyl)piperazin-1-yl]ethanesulfonic acid; PBS, phosphate buffered saline; PET, polyethylene terephthalate; PC, polycarbonate; PTFE, polytetrafluoroethylene; PMT, photomultiplier tube; HyD, GaAsP hybrid detector; f-CARS, forward CARS; epi-CARS, backward scattered CARS; OPO, optical parametric oscillator; BF, bright field; Calcein AM, acetoxymethyl derivate of calcein; EthD-1, ethidium homodimer; ER, endoplasmic reticulum; SNEDDs, self-(nano)-emulsifying drug delivery systems; NLCs, nanostructured lipid carriers; SLNs, solid lipid nanoparticles.

* Corresponding author.

E-mail addresses: jukka.saarinen@helsinki.fi, saarinenjukka88@gmail.com (J. Saarinen). pharmaceutical sciences, about 300 publications have been published annually since 2010. When cultured with suitable media and membrane inserts, these cells grow as monolayers on membranes and express morphology with many features in common with the intestinal epithelium, including tight junctions, carrier proteins and microvilli (Artursson et al., 2001; Hubatsch et al., 2007). Typically, it takes approximately 21 days until this monolayer is mature and confluent. The culture is then used in drug permeation studies during which a drug is introduced to one side of the insert and the amount of drug having crossed the cell layer to the other side of the membrane is measured at defined time points (Artursson et al., 2001; Artursson, 1990). Technically, the procedure is simple, but direct information on the cell layer composition and structure during the growth period is not obtained. Furthermore, laboratories can have slightly different methods of culturing Caco-2 cells and it is recognized that different culturing conditions (cell passage number, initial seeding density, transport buffer) can lead to poor reproducibility and significantly variable results in drug transport studies (Donna, 2008; Hubatsch et al., 2007). However, the reasons and mechanisms for these differences are often not known. 
Visualization of the live cells would help to address this analytical shortcoming and could facilitate understanding of structural sources of drug permeation testing variability. Confocal fluorescence microscopy has been widely used to image cells, including Caco-2 cells. The use of fluorescence microscopy has made it possible to probe virtually any part of a cell culture with high specificity using fluorophores specific to various cellular components. Lipid droplets have recently been recognized as important dynamic cellular components that, in addition to acting as energy storage, can possess other dynamic cellular functions and, as a result, lipid droplet studies are gaining widespread attention (Martin and Parton, 2006). Lipid droplets can be found in high numbers in adipose cells, although most cell types contain lipid droplets to varying degrees (Pol et al., 2014; Tauchi-Sato et al., 2002). These lipid droplets can be visualized using fluorescence microscopy and new fluorophores have recently been developed for this purpose (Wang et al., 2016). However, there are some drawbacks to using externally added fluorophores. Firstly, at least one extra sample preparation step is required. Secondly, these agents do not naturally belong in cells and it is often not known how they affect the cellular functionality. Thirdly, bleaching of the fluorescent labels may render prolonged time-lapse experiments impossible. Thus, alternative imaging techniques that are labelfree and chemically-specific are needed.

Coherent anti-Stokes Raman scattering (CARS) microscopy is a label-free imaging approach based on molecular vibrational resonances that is attractive for imaging biological samples, including live cell cultures (Evans and Xie, 2008; Winterhalder and Zumbusch, 2015). In the CARS process, three pulsed lasers are overlapped spatially and temporally at the focus of a high numerical aperture (NA) objective. The initiated non-linear four-wave mixing process generates a strong anti-Stokes Raman signal when matched with the vibrational resonances of molecule within the focal volume of the sample. This enables rapid, label-free, and chemically-specific imaging with (sub-)micron spatial resolution.

CARS microscopy has been used to image various cellular components including the cytosol and nucleus (Bonn et al., 2009; Cheng et al., 2002; El-Mashtoly et al., 2014) and, in particular, lipid structures. Cells and tissues are rich in lipid structures, such as membrane structures and lipid droplets. Lipids have a high $\mathrm{CH}$ bond density and the highly CARS-active $\mathrm{CH}$ stretching modes in the CARS spectral region of $2800-3000 \mathrm{~cm}^{-1}$ can be exploited to visualize lipid structures (Bonn et al., 2009; Evans et al., 2005; Nan et al., 2006; Wong et al., 2011). Hellerer et al. have also shown the benefit of using CARS for visualization of lipid droplets compared to fluorescence imaging (Hellerer et al., 2007). In their study it was shown that the number of lipid droplets in live Caenorhabditis elegans nematode was underestimated when stained lipid droplets (Nile red stain) were imaged using two-photon fluorescence microscopy as compared to CARS microscopy where the signal originates from the molecular vibrations of carbon-hydrogen bonds.

In the present study, the aim was to investigate the lipid content and change in live Caco- 2 cells cultured as monolayers on membrane inserts. The lipid content and distribution is of high importance because these cells have been routinely used in drug intestinal permeation studies and the lipid content could strongly affect the permeation of drugs through these cells. Replication of drug permeation between laboratories has been problematic, which may be partly due to unrecognized differences in lipid content and distribution.

To the best of our knowledge, there are no previous studies reporting label-free CARS imaging of live intestinal epithelial cell cultures grown on membrane inserts. An in vitro imaging platform using CARS microscopy to study live intestinal epithelial cell cultures could be used to obtain structural information about the cell cultures. Therefore, in the present study, we have investigated the potential of label-free CARS microscopy for non-destructive imaging of live intestinal epithelial cell cultures in (bio)pharmaceutically relevant conditions and have then used the technique to obtain new insights into Caco-2 cell structures. Herein, we investigated the most suitable buffer types, membrane insert materials, and imaging conditions for CARS imaging of cell cultures and demonstrated that CARS microscopy is suitable for label-free and non-destructive imaging of live Caco-2 cells, including the analysis of lipid structures.

\section{Materials and methods}

\subsection{Cell culture materials}

Cell culture medium solution Dulbecco's Modified Eagle's Medium (DMEM), fetal bovine serum (FBS), L-glutamine, nonessential amino acids (NEAA), Penicillin ( $100 \mathrm{IU} / \mathrm{mL}$ ), Streptomycin

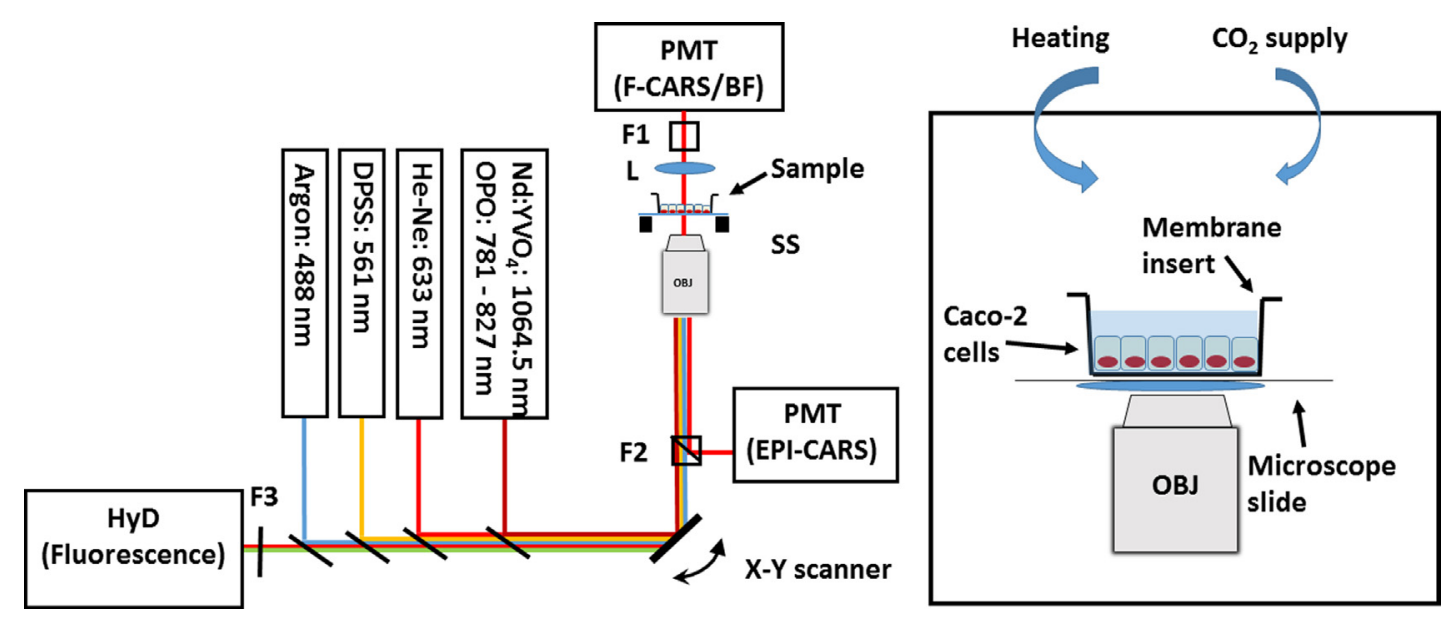

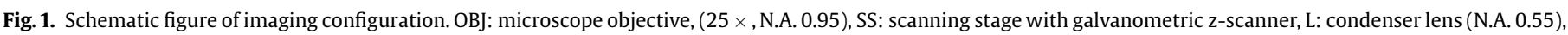

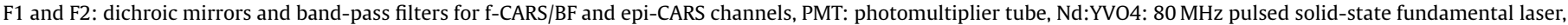

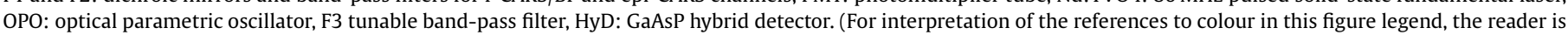
referred to the web version of this article.) 
( $100 \mathrm{mg} / \mathrm{mL}$ ) and trypsin were purchased from HyCLone, USA. Ethylenediaminetetraacetic acid disodium salt dihydrate (EDTA) and 2-[4-(2-hydroxyethyl)piperazin-1-yl]ethanesulfonic acid (HEPES) were acquired from Sigma-Aldrich, Germany. Hank's balanced salt solution (HBSS) and phosphate buffered saline (PBS) were purchased from Life Technologies, USA. The $25 \mathrm{~cm}^{2}$ and $75 \mathrm{~cm}^{2}$ culture flasks and Transwell ${ }^{\circledR}$ inserts made from polyester (polyethylene terephthalate, PET) with a pore size of $3.0 \mu \mathrm{m}$, polycarbonate (PC) with a pore size of $3.0 \mu \mathrm{m}$, and collagen coated polytetrafluoroethylene (PTFE) with pore sizes of $3.0 \mu \mathrm{m}$ and $0.4 \mu \mathrm{m}$, were obtained from Corning Inc., USA. Human colon carcinoma cells (Caco-2) were obtained from the American Type Culture Collection (ATCC), USA. The glass bottomed 24-well plates that were used in the viability tests were obtained from MatTek Corporation, USA.

\subsection{Cell culture protocol}

Caco-2 cells were cultured in 25 or $75 \mathrm{~cm}^{2}$ culture flasks in DMEM with $10 \%(\mathrm{v} / \mathrm{v})$ FBS, $1 \%(\mathrm{v} / \mathrm{v}$ ) L-glutamine, $1 \%(\mathrm{v} / \mathrm{v})$ NEAA and $1 \%(\mathrm{v} / \mathrm{v})$ antibiotic mixture (final concentration of $100 \mathrm{IU} / \mathrm{mL}$ Penicillin and $100 \mathrm{mg} / \mathrm{mL}$ Streptomycin). Cell passage numbers 3139 were used. The cells were passaged once a week. 0.5 mM PBSEDTA and $0.25 \%(\mathrm{w} / \mathrm{v})$ trypsin-PBS-EDTA solutions were used for passaging the cells. For imaging experiments, the cells were seeded on Transwell ${ }^{\mathbb{R}}$ inserts with an initial density of 60,000 cells per $1 \mathrm{~cm}^{2}$. Cells were cultured at $37^{\circ} \mathrm{C}$ in a humidified atmosphere (95\%) with $5 \% \mathrm{CO}_{2}$.

\subsection{Microscope configuration}

The microscope used for imaging was a commercially available fully-integrated Leica TCS SP8 CARS microscope. A heated environmental chamber built over the microscope with a controlled $\mathrm{CO}_{2}$ supply $\left(37^{\circ} \mathrm{C}\right.$ and $5 \% \mathrm{CO}_{2}$ ) was used for maintaining the live cell cultures during imaging. The system consists of an inverted microscope equipped with a laser-scanning confocal scan-head and photomultiplier tube (PMT) and GaAsP hybrid (HyD) photodetectors (Fig. 1). The CARS signal was separated from other signal components (e.g. second harmonic generation, SHG) using bandpass filters at $560-750 \mathrm{~nm}$ and detected in the forward direction (f-CARS) or backward direction (epi-CARS) using nondescanned PMT detectors. A water-immersion $25 \times$ objective with an NA of 0.95 (Leica HCX IR APO L $25 \times / 0.95 \mathrm{~W}$ ) was used in all experiments. The CARS excitation source was a $\mathrm{Nd}: \mathrm{YVO}_{4}$ solidstate-laser (APE GmbH, Germany) with an optical parametric oscillator (OPO). The Stokes beam $\left(\omega_{\mathrm{s}}\right)$ had a fixed wavelength of $1064.5 \mathrm{~nm}$ and a pulse duration of $7 \mathrm{ps}$. The bandwidth was about $2-3 \mathrm{~cm}^{-1}$ and the repetition rate was $80 \mathrm{MHz}$. The pump and probe beams $\left(\omega_{\mathrm{p}}\right.$ and $\left.\omega_{\mathrm{pr}}\right)$ at $781-827 \mathrm{~nm}$ were generated from the OPO with the pulse duration of 5-6 ps. Experiments were performed with laser powers of up to $100 \mathrm{~mW}$ for the pump/probe beam and $50 \mathrm{~mW}$ for the Stokes beam, corresponding to a maximum peak power density of $\sim 0.14 \mathrm{TW} / \mathrm{cm}^{2}$ within the diffraction limited spot. The incident powers were measured by a photodiode power sensor (S170C, Thorlabs Inc., USA). The effective beam areas were calculated as (Eq. (1))

$A_{e f f}=\pi w^{2} / 2$,

where $w$ is the $1 / \mathrm{e}^{2}$ beam radius of the Gaussian fit to the Airy disk $(w=0.687 \mathrm{r}$; Rayleigh limit $r=0.61 \lambda / \mathrm{NA}$, where $\lambda$ is the excitation wavelength).

For the bright field light microscopy (BF) imaging a He/Ne laser with a wavelength of $633 \mathrm{~nm}$ and power below $0.1 \mathrm{~mW}$ was used. The Leica Application Suite Advanced Fluorescence (LASAF) was used for image acquisition and processing together with Fiji Image J software. LASAF was used to estimate the diameter of individual lipid droplets in cells. Contrasts were adjusted case-by-case in images.

\subsection{CARS spectroscopy of solution media}

CARS spectra from DMEM with and without phenol red, HBSS, and PBS were measured to find the optimal media solution for imaging. A drop of each solution was placed on glass coverslips which were then scanned in the yz-plane in order to acquire CARS signal from both the sample and the coverslip. The spectra were recorded from $2698 \mathrm{~cm}^{-1}$ to $3410 \mathrm{~cm}^{-1}$ by tuning the pump laser step-by-step 71 times during a spectral scan. The sample spectra were normalized to the spectrum of the coverslip. Since glass only generates a nonresonant CARS signal, it was possible to cancel out the intensity variations due to the laser power fluctuations and the wavelength dependent detection efficiency.

\subsection{Imaging of membrane inserts}

The durability of different membrane insert materials to CARS lasers and the optical properties of inserts were evaluated to find the best insert for CARS imaging. Commonly available Transwell ${ }^{\mathbb{R}}$ inserts (Corning Inc., USA) made from three different materials, PET, PC and collagen-coated PTFE, were imaged. The inserts were placed on a coverslip while being imaged. Z-stacks covering the entire thickness of the insert membrane were acquired at $2860 \mathrm{~cm}^{-1}$ with increasing laser powers until visible damage was observed. Inserts were imaged dry as well as after the addition of HBSS-HEPES buffer solution on the apical side to see if the buffer changed the optical behavior of the insert materials and image quality. Bright field images of the inserts were taken before and after CARS imaging.

\subsection{Cell viability analysis}

The effect of CARS imaging on cell viability was investigated at $37{ }^{\circ} \mathrm{C}$ and $5 \%$ of $\mathrm{CO}_{2}$. The cells were seeded on glass-bottomed $24-$ well plates at a density of approximately 325000 cells/well and incubated overnight to allow the cells to attach to the bottom of the wells. The cells were imaged using CARS microscopy the next day. Cells were exposed to the CARS lasers at different powers and for different durations. Bright field images were recorded before and after exposure to the lasers to detect any changes in cellular morphology.

To attain further information about the effect of laser irradiation on cell viability, a fluorescence assay was performed using the CytoSelect ${ }^{\mathrm{TM}}$ Cell Viability and Cytotoxicity Assay Kit (Cell Biolabs, Inc., USA, Catalog Number CBA-240). The kit contains an acetoxymethyl derivate of calcein (Calcein AM) and ethidium homodimer (EthD-1) fluorescence dyes. Live cells convert the nonfluorescent Calcein AM to the highly fluorescent calcein, which remains within the cells. EthD-1 is a fluorescent stain that can only penetrate damaged cell membranes and fluoresces strongly when bonded to ssDNA, dsDNA, RNA, oligonucleotides and triplex DNA. Thus, it was used to detect dead cells. Before performing the assay, CARS z-stacks with different laser powers $(20-100 \mathrm{~mW}$ for the pump beam and $10-50 \mathrm{~mW}$ for the Stokes beam in the focus) were recorded of the Caco-2 cells. Z-stacks were recorded so that they covered the entire thickness of the cells in the field of view. The number of steps in the z-stack was 45 and the step size was $0.57 \mu \mathrm{m}$. Images with $512 \times 512$ pixels were recorded using a $2 \times$ zoom, producing a scanning area of $232 \times 232 \mu \mathrm{m}^{2}$. A scanning speed of $400 \mathrm{~Hz}$ (line average 2) was used, resulting a pixel dwell time of $1.20 \mu \mathrm{s}$ and a frame rate of 0.386 frames/s. After recording 
each CARS stack, the medium was removed from the wells and $400 \mu \mathrm{L}$ of fluorescence dye containing Calcein AM and EthD-1 (diluted in HBSS-HEPES, 1:500) was added per well. The cells were then incubated for $30 \mathrm{~min}$ at $37^{\circ} \mathrm{C}$. After incubation, the excess stain was removed by washing the cells twice with HBSS-HEPES buffer solution. The cells were kept in buffer while imaging the cell viability. HyD-detectors were used for imaging the green fluorescence signal from calcein and the red fluorescence signal from EthD-1. For the negative control, the cells were incubated with HBSS-HEPES buffer solution without exposure to the CARS lasers. For the positive controls indicating dead cells, the cells were incubated with Saponin (diluted in HBSS-HEPES, 1:100) or $1 \%$ Triton-X 100 in HBSS-HEPES for $10 \mathrm{~min}$ and imaged afterwards in the same manner as the samples that were exposed to different CARS laser powers.

\section{Results and discussion}

\subsection{CARS spectra of the media solutions}

Despite the importance of selecting suitable cell culture media solutions for CARS imaging, published reports in this area are lacking. CARS spectra of different media solutions were recorded to find the solution that gives the lowest background CARS signal. Four media solutions for intestinal epithelial cell culturing were tested in this study: two buffer solutions commonly used for cell experiments, PBS and HBSS-HEPES, and the cell culturing medium DMEM, with and without phenol red.

The CARS spectra of the four media are similar within the spectral region analysed (Fig. 2). The broad peak centered at $\sim 3200 \mathrm{~cm}^{-1}$, common to all spectra, is associated with stretching of the - $\mathrm{OH}$ group of water. In addition to water, PBS consists of inorganic salts such as $\mathrm{KH}_{2} \mathrm{PO}_{4}, \mathrm{NaCl}$ and $\mathrm{Na}_{2} \mathrm{HPO}_{4}$, whose Raman active modes are outside the CARS spectral region probed. HBSS also contains $\mathrm{KCl}$ with the addition of HEPES. Sulfonic acid within the HEPES, has a Raman signal at $2936 \mathrm{~cm}^{-1}$ due to $-\mathrm{OH}$ stretching (Panicker et al., 2006). However, no such peak was distinguishable in the present study; presumably it was masked by the broader and stronger water signal. DMEM is a culture medium widely used for cell culturing. DMEM contains glucose and, in this study, FBS, L-glutamine, NEAA and an antibiotic mixture of Penicillin and Streptomycin. However, the concentrations of these

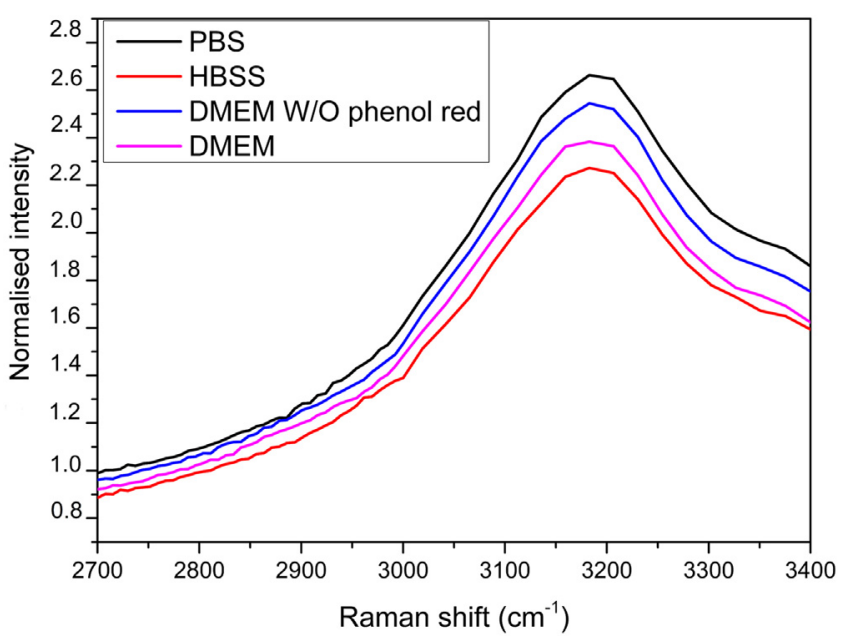

Fig. 2. CARS spectra of four different media solutions: PBS, HBSS-HEPES and DMEM with and without phenol red. (For interpretation of the references to colour in this figure legend, the reader is referred to the web version of this article.) species are evidently too low for the signals from these species to be detectable.

The region at around $2800-3000 \mathrm{~cm}^{-1}$ is interesting for imaging cells, because various - $\mathrm{CH}$ stretching modes occur in this region. Thus, for CARS imaging it is preferable to use a solution that does not contain species that exhibit strong Raman scattering in this region. Overall, the CARS signal strength was weak and no major differences in the CARS spectra could be seen between the media solutions. Thus, all the media are suitable for imaging cell cultures in this spectral region. In this study, HBSS-HEPES was subsequently used while cells were imaged on membrane inserts.

\subsection{Imaging membrane inserts}

The durability of different insert materials to the CARS lasers was evaluated by recording $z$-stacks of the membranes (without buffer present). Fig. 3a shows a bright field image after recording a $z$-stack across the entire thickness of a PC membrane $(\sim 22 \mu \mathrm{m})$ with laser powers of $32 \mathrm{~mW}$ and $14 \mathrm{~mW}$ for the pump and Stokes beams, respectively. Obvious damage, manifested as holes and surrounding dark regions, is visible. Such damage occurred when laser powers as low as $15 \mathrm{~mW}$ for the pump and $7 \mathrm{~mW}$ for the Stokes beam were focused on the filters. The ongoing damage was also seen as a broadband luminescence emission on all PMT detector channels. The damaged areas are expected to contain carbon (graphite) particles. These particles absorb light through one-photon and two-photon excitation which causes fluorescence over a broad spectral range. Part of this emission falls into the detection range of the CARS channel and is seen as an intense signal (Fig. 3b). Strong absorption could possibly trigger further thermal damage on the membrane. Similar behavior was observed when PET inserts were imaged. When a laser power of $47 \mathrm{~mW}$ for the pump and $20 \mathrm{~mW}$ for the Stokes were used, even one scan $(1024 \times 1024$ pixels $)$ was sufficient to damage the PET inserts (Fig. 4f). In contrast, the PTFE inserts showed superior durability compared to the PC and PET inserts. It was possible to record CARS z-stacks with maximum laser powers with no apparent optical damage.

The effect of HBSS-HEPES addition on the optical properties and durability of the inserts was also investigated. The pores of PC and PET inserts (with a pore size of $3 \mu \mathrm{m}$ ) were clearly visible as dark spots with BF imaging (Fig. $4 \mathrm{a}-\mathrm{b}$ and d-e). Unlike for the PC and PET inserts, the pores at the surface of the PTFE inserts were not visible in the BF or CARS images (Fig. $4 \mathrm{~g}-1$ ). Instead, a meshworklike structure was visible. An increase in signal strength during CARS imaging was seen with all the inserts after addition of the HBSS-HEPES buffer (CARS images without HBSS-HEPES are not shown due to the lack of sufficient signal to be detected). With the collagen coated PTFE inserts, a clear change in BF images could also be seen (Fig. $4 \mathrm{~g}-\mathrm{h}$ and $\mathrm{j}-\mathrm{k}$ ). The surface of the inserts appeared to be smoother in the presence of the solution (Fig. $4 \mathrm{~g}-\mathrm{h}$ and j-k). These PTFE-inserts are coated with an equimolar mixture of types I and III collagen (Corning Inc., Product \#3491 and 3492), and the visible meshwork structure is probably due to the arrangement of the collagen fibers on top of the insert membrane. This meshwork was not obvious in the CARS images when dry inserts were imaged. However, better refractive index matching after addition of HBSSHEPES buffer solution made it visible when relatively high laser powers were used.

The addition of HBSS-HEPES did not improve the durability of PC and PET inserts. When the lasers were not focused on these membranes, damage was generally not apparent, and thus they could potentially be used in cell culture experiments with CARS imaging if care was taken never to focus on the membranes. However, when imaging the Caco-2 cell cultures through these filter membranes, the shadows of the scattering pore pattern 


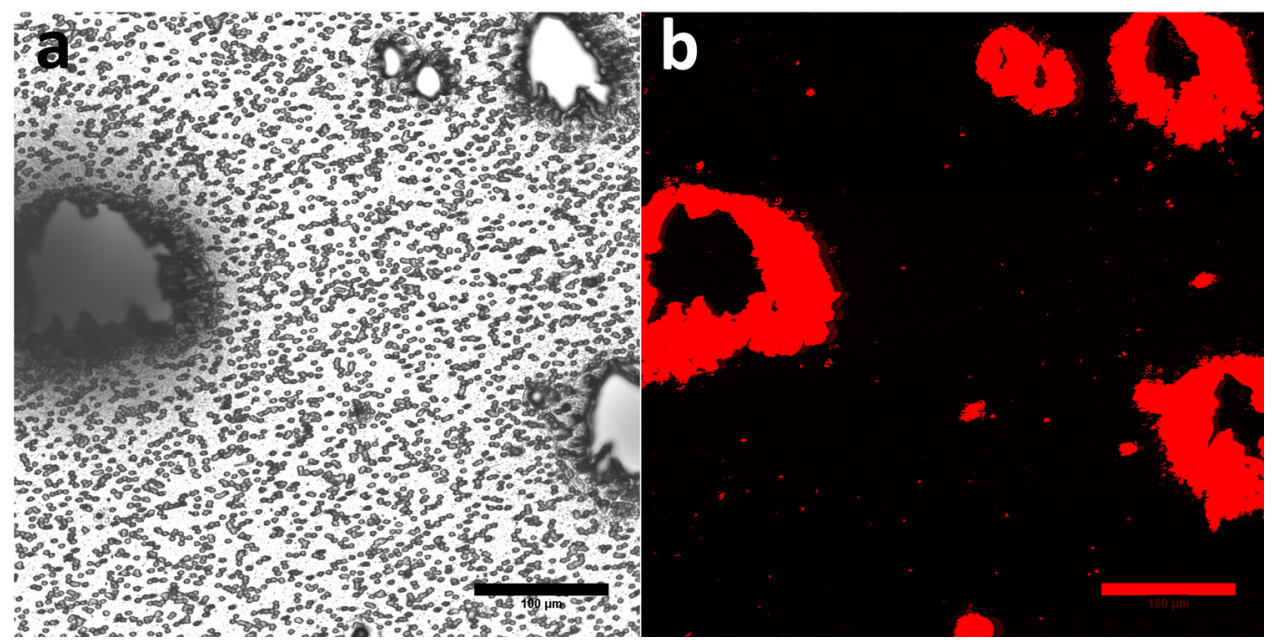

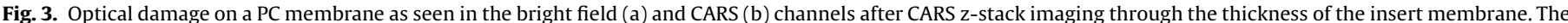

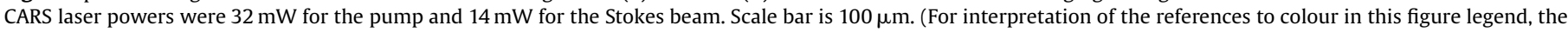
reader is referred to the web version of this article.)

obscured the cells (Fig. 5). Thus, it can be concluded that PTFE is the best insert material for CARS imaging of cell cultures as a result of its high durability to laser irradiation and high transparency. The cell culture images obtained when PTFE inserts were used are shown in Section 3.4.

\subsection{Cell viability after CARS imaging}

In general, the CARS process is thought to be rather well tolerated for cell and tissue imaging (Evans et al., 2005; Evans and Xie, 2008) and CARS microscopy is useful for long-term live cell microscopy studies (Jüngst et al., 2013). The CARS process itself occurs from the ground electronic state, which reduces photodamage (Evans and Xie, 2008). In addition, many CARS microscopes use picosecond pulsed lasers, which have a lower peak power compared to femtosecond lasers that are typically used in two-photon fluorescence (Cheng et al., 2002; Evans and Xie, 2008). However, multi-photon absorption induced photodamage can still occur when Stokes and pump lasers are irradiated onto the sample during CARS imaging. Thus, it is important to confirm the viability of the cells in order to be able to image cells that remain alive and healthy under the experimental time needed. Since there are different CARS setups between research groups it is not trivial to compare the photodamage observed in various studies unless all variables are accounted for. These variables include laser wavelength(s), pulse width, and repetition rate, objectives used and image acquisition time including pixel dwell time and scanning speed.

In this study, the changes in the morphology of the cells after CARS image acquisition were initially examined based on the BF images before and after exposure to the CARS lasers as described in Section 2.3. No major changes in cell morphology were observed. After exposing the cell culture for $5 \mathrm{~min}$ with powers of $100 \mathrm{~mW}$ in the pump and $50 \mathrm{~mW}$ in the Stokes beam (total irradiance of $\sim 0.14 \mathrm{TW} / \mathrm{cm}^{2}$ ) it was possible to see some changes in cell culture structure: the cells were less tightly associated with one another (data not shown), indicating photodamage. This result is in accordance with previous studies where comparable irradiance values were used and is attributed to the chemical changes due to formation of free electrons in the sample (Galli et al., 2014; Vogel et al., 2005). However, these were extreme conditions that are not generally needed for cell imaging. Wong et al. showed that there was apparent photodamage in the $\mathrm{BF}$ image of $\mathrm{NIH} / 3 \mathrm{~T} 3$ fibroblast cells after CARS z-stack imaging with laser powers of $\sim 35 \mathrm{~mW}$
(Wong et al., 2011). The same cell was also imaged after $2 \mathrm{~h}$ and it had altered its morphology to become round-shaped and was detached from the surface of the Petri dish, indicating cell death. In our case, no such effects were observed when Caco-2 cells were imaged using z-stacks and pump and Stokes laser powers of $40 \mathrm{~mW}$ and $20 \mathrm{~mW}$, respectively. The bright field images were recorded before and two hours after the CARS imaging. After two hours the cells were still attached to the bottom of the 24-well plate and no change in morphology was observed. In addition to instrumental conditions, it is likely that different cell types and the structure of their cultures exhibit different optical sensitivities. For example, Caco- 2 cells tend to associate closely with one another, unlike NIH/3T3 fibroblasts (Wong et al., 2011).

Since it is difficult to evaluate the viability of the cells just by their morphology, fluorescence imaging was also performed. Fluorescent stains were used to selectively stain live and dead cells, with Calcein AM used to stain the live cells and EthD-1 used to stain the dead cells, as described in Section 2.6.

The results of the cell viability study are shown in Fig. 6. After the full z-stack ( $\sim 2$ min exposure time) at laser powers of $100 \mathrm{~mW}$ and $50 \mathrm{~mW}$ for the pump and Stokes beams respectively, the cell membranes were not sufficiently damaged for EthD-1 to contact the DNA-like structures and fluoresce. Thus, according to this analysis, the Caco- 2 cells were still viable soon after CARS image acquisition even with the high laser powers. However, one has to be careful when prolonged experiments with live cells are conducted, since photodamage can also result after extended cell stress (Magidson and Khodjakov, 2013). Furthermore, more subtle cell damage may need to be taken into account. For example, lipid droplet trafficking or other internal cellular processes could be disrupted with lower laser powers and energies. Thus, it is best to keep the laser powers as low as possible, while still being able to capture images of sufficient quality. Nan et al. imaged lipid droplet transport in living steroidogenic mouse adrenal cortical (Y-1) cells using CARS microscopy, and concluded that laser pulse energies should be under $2 \mathrm{~nJ}$ with an average power under $9 \mathrm{~mW}$ to be able to image lipid droplet transport nondestructively (Nan et al., 2006). They used shorter wavelengths in their setup: $711 \mathrm{~nm}$ and $892 \mathrm{~nm}$ for the pump and Stokes respectively, compared to the present study, in which $816 \mathrm{~nm}$ and $1064.5 \mathrm{~nm}$ for the pump and Stokes were employed. Photodamage can be reduced with the use of longer excitation wavelengths (Fu et al., 2006).

Hopt and Neher have studied photodamage in cells during twophoton fluorescence microscopy (Hopt and Neher, 2001). They 


$\begin{array}{cc}\text { BF, } & \text { BF, } \\ \text { dry insert } & \text { insert with } \\ \text { HBSS-HEPES }\end{array}$

\section{CARS, insert with HBSS-HEPES}

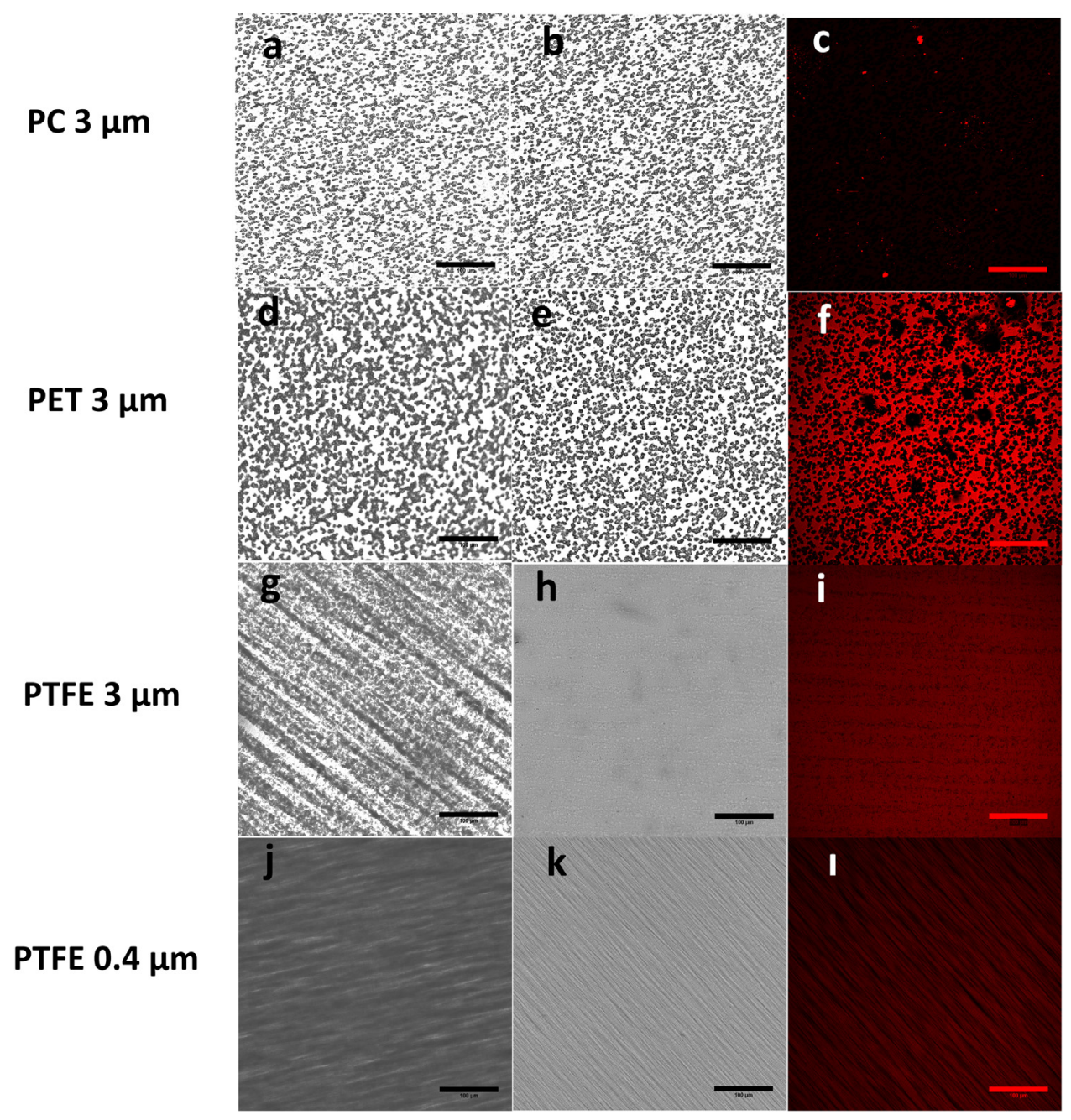

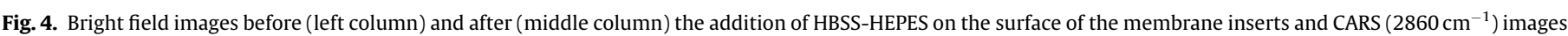

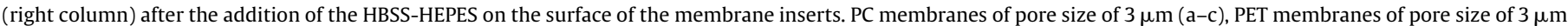

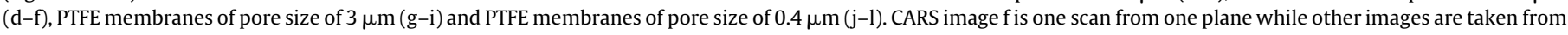

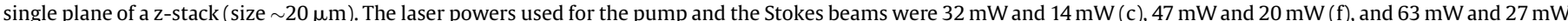

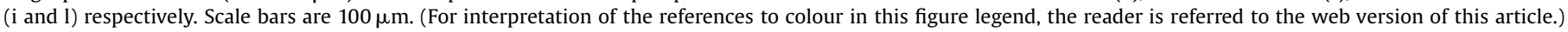

used bovine adrenal chromaffin cells that were exposed to repeated laser scans with varied beam and scan parameters including average light intensity, pulse duration, excitation volume and scan speed while having a pulse width of $190 \mathrm{fs}$, a wavelength of $840 \mathrm{~nm}$ and a pulse repetition rate of $82 \mathrm{MHz}$ as default parameters. They observed cell damage based on morphological changes and the $\mathrm{Ca}^{2+}$-indicator dye, FURA-2, which suddenly loses its intensity due to a higher amount of $\mathrm{Ca}^{2+}$ in the damaged cells. Based on these repeated scans, they formulated the following equation to predict the threshold limit for how many scans cells can be exposed to using ultrafast near-infrared lasers before photodamage occurs (Eq. (2)):

$$
N \propto \frac{(f \tau)^{1.5}}{t_{d}\langle P(t)\rangle^{2.5}}
$$

where $N$ is the number of scans, $f$ is the repetition rate, $t_{d}$ is the pixel dwell time and $P$ is the average power. If this equation is used with the extreme conditions in the viability study above, when the laser pulse width was $\sim 5$ ps and the total average power is $150 \mathrm{~mW}$, the number of scans needed to produce photodamage is almost 800 . In the present viability studies, the number of scans used for one z-stack was much smaller (45 z-levels), suggesting that damage would not occur. It should also be kept in mind that when z-stacks are recorded the excitation plane changes and laser exposure is distributed over a larger volume in the cells. Thus, zstacks are likely to be less damaging to cells compared to repeated scans on the same plane.

In this study, we have demonstrated that using a pixel dwell time of $1.2 \mu \mathrm{s}$, frame rate of $2.59 \mathrm{~s}$ per frame, 45 scans per $\mathrm{z}$-stack, pulse repetition rate of $80 \mathrm{MHz}$, pulse width of $5-7 \mathrm{ps}$ and $1064.5 \mathrm{~nm}$ and $816 \mathrm{~nm}$ laser wavelengths, no damage to the cells 


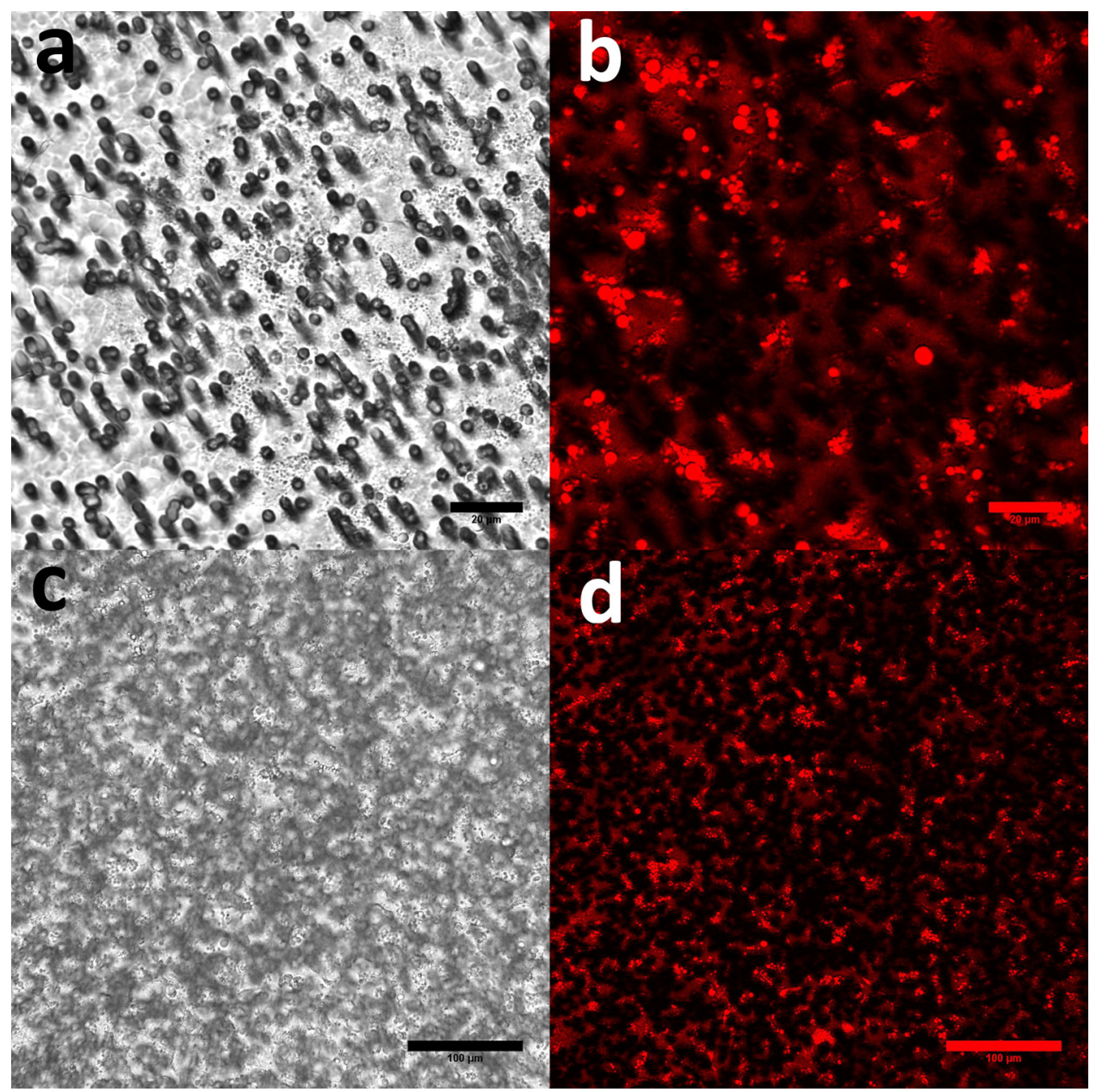

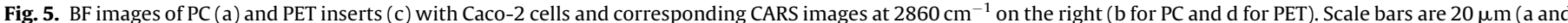
b) and $100 \mu \mathrm{m}$ (c and d). (For interpretation of the references to colour in this figure legend, the reader is referred to the web version of this article.)

was apparent even with a total average power as high as $150 \mathrm{~mW}$. Typically, total average powers of $18-32 \mathrm{~mW}$ for pump/probe beam and $8-14 \mathrm{~mW}$ for the Stokes beam in the focus were needed to produce good quality images of lipid structures in Caco-2 cells. These settings were subsequently used for imaging Caco-2 cells grown on PTFE inserts.

\subsection{Imaging of the Caco-2 cells grown on PTFE inserts}

After screening the most suitable cell culturing and imaging conditions including membrane insert materials, buffer solutions and laser powers for label-free non-destructive imaging of Caco-2 cells on membrane inserts, the second main objective of this study was to use CARS microscopy to reveal new structural information about Caco-2 cell cultures over the typical culture period of 21 days. During this time the Caco- 2 cell cultures usually reach sufficient integrity and maturity to be used as in vitro models in drug permeation studies (Shah et al., 2006). First, CARS spectra in the $\mathrm{CH}$ stretching region were measured. A typical spectrum of a lipid droplet and another region with lower signal intensity at $2860 \mathrm{~cm}^{-1}$ in the centre of a cell are shown in Fig. 7a. In the lipid droplets, the strongest CARS signal occurred at around $2860 \mathrm{~cm}^{-1}$ $\left(\mathrm{CH}_{2}\right.$ stretching). In addition, a shoulder occurred in the spectrum above $2900 \mathrm{~cm}^{-1}$ ( $\mathrm{CH}_{3}$ stretching) (Volkmer et al., 2001). The $\mathrm{CH}_{2}$ stretching mode has been used to image lipid-rich cellular structures, since this moiety is more concentrated in lipid based structures than other chemical moieties (Evans and Xie, 2008; Garrett et al., 2012; Lin et al., 2011). No strong CARS peaks were observed in the spectrum that was measured in the centre of the cell. Above $3000 \mathrm{~cm}^{-1}$ there is a broad peak in both spectra due to the aqueous environment.

As mentioned, lipid droplet studies are gaining interest and more information about the cellular functions of lipid droplets is being obtained. In general, the lipid droplet size can vary substantially depending on the cell type and, for example, adipocytes can have a single lipid droplet tens or hundreds of microns in size (Thiam et al., 2013). A phospholipid monolayer forms the outer surface of the lipid droplets, and the core consists mainly of neutral triacylglycerol and cholesterol esters (Murphy, 2001). It is not completely understood how intracellular lipid droplets are formed, although they are believed to originate from the endoplasmic reticulum (ER). Neutral lipids are synthesized inside the bilayer of the ER from where lipid droplets with the phospholipid monolayer surface bud after maturation (Martin and Parton, 2006; van Meer, 2001). Lipid droplets are seen nowadays as dynamic organelles that also contain and interact with many proteins (Martin and Parton, 2006; Salo et al., 2016). Intralipid droplet proteins of particular importance are the perilipin family proteins, which regulate the balance between the degree of lipid storage and hydrolysis of stored triacylglycerols (Ducharme and 


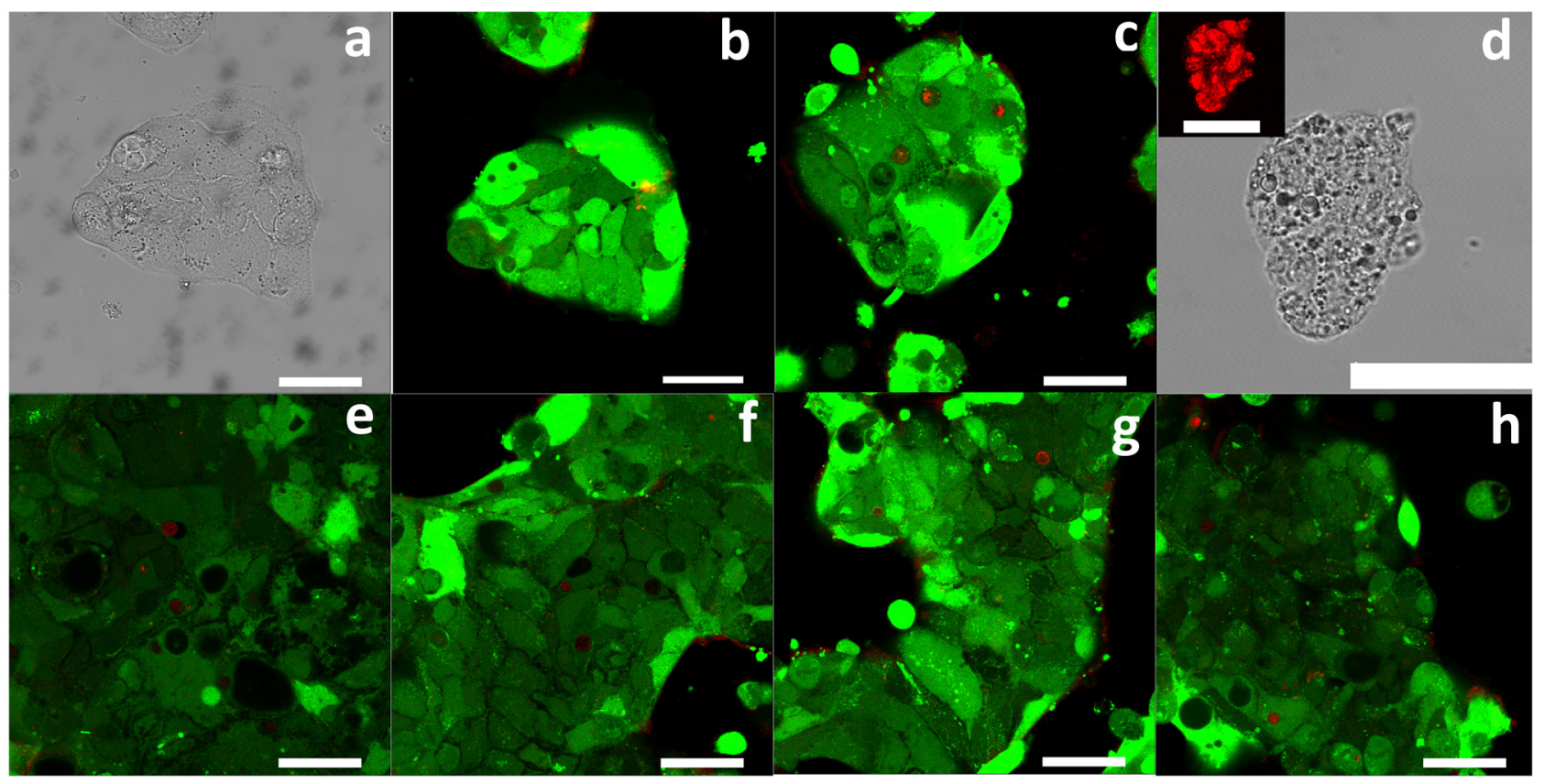

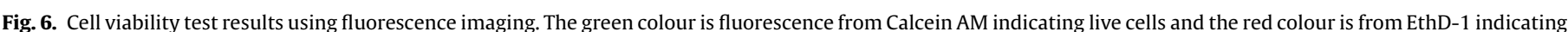

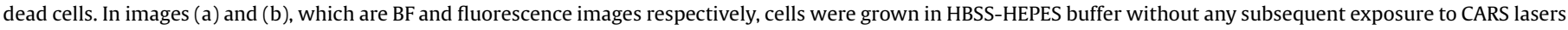

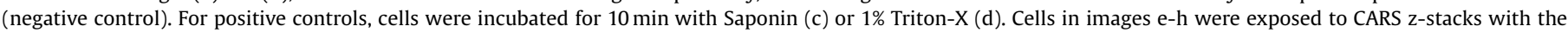

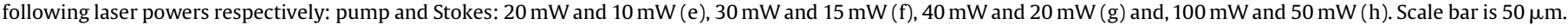
(For interpretation of the references to colour in this figure legend, the reader is referred to the web version of this article.)

Bickel, 2008). In addition, intracellular lipid droplet accumulation has been recognized in several diseases, including diabetes (Ducharme and Bickel, 2008) and atheroschlerosis (Paul et al., 2008).

The structural evolution of the lipid-rich structures in Caco-2 cells was evident during the cell culture progression. After 7 days of culturing many small lipid droplets were visible in the CARS images (Fig. 7b). The size of the lipid droplets increased substantially over the 21-day period. After 14 days of culturing the diameter of the largest lipid droplets was $\sim 7 \mu \mathrm{m}$ (Fig. 7c). The diameter of a Caco-2 cell is $\sim 20 \mu \mathrm{m}$, meaning that in these cases over $10 \%$ of the cellular cross section was filled with a single lipid droplet.

Human colon cancer tissue has an increased number of lipid droplets compared to healthy tissue (Accioly et al., 2008). Since Caco-2 cells are colon adenocarcinoma cells, these cells may contain larger quantities of lipid droplets than would be expected in healthy tissue in vivo. Accioly et al. used electron microscopy to show that Caco-2 cells have a 4 to 5 -fold increase in the number of lipid droplets compared to rat intestinal epithelial cells (IEC-6 cells) after $24 \mathrm{~h}$ of culturing. Scalfi-Happ et al. used confocal spontaneous Raman microscopy for imaging Caco- 2 cells and IEC- 6 cells and concluded that Caco-2 cells had substantially higher lipid droplet accumulation compared to non-malignant IEC-6 cells (Scalfi-Happ et al., 2011). Our results support these findings. However, to the best of our knowledge, the present study is the first where live unlabelled Caco- 2 cells have been imaged in a (bio) pharmaceutically relevant environment for drug permeation studies, including on membrane inserts. It is more demanding to capture high quality images in these conditions compared to situations where cells have been grown on glass slides or glassbottomed well-plates, which are typically used for CARS imaging. As demonstrated here, the membrane insert material can have a great impact on the image quality of the cells. We have shown here that after optimization of the imaging conditions, live intestinal epithelial cells grown on membrane inserts can be efficiently imaged using CARS microscopy.

As mentioned, Caco-2 cells are frequently used as an in vitro intestinal cell model in studies predicting drug transport across the intestinal epithelium. However, with many drugs being hydrophobic and/or lipophilic, the lipid content of the cells will influence drug permeation. Therefore, knowledge of lipid content and distribution in exactly the same cell cultures that are used for drug permeation studies may help address the reasons for the wide variability in drug permeation observed between laboratories with these cultures. CARS microscopy allows for such analysis.

Furthermore, different types of lipid based drug delivery systems, such as solid lipid nanoparticles (SLNs), nanostructured lipid carriers (NLCs) and self-(nano)-emulsifying drug delivery systems (SNEDDSs) have been used to enhance intestinal absorption of hydrophobic drugs (Chai et al., 2016; Li et al., 2016; Neves et al., 2016). The mechanisms of improved drug absorption using these drug delivery systems at the cellular level are not still fully understood. Nevertheless, the interactions between these formulations and cells may lead to uptake of intact particles via endocytosis (and therefore enhance trans-epithelial drug delivery), or lipid-mediated alteration of tight junction integrity (increasing paracellular drug delivery) (Chai et al., 2016; Li et al., 2016; Neves et al., 2016). CARS microscopy could be used to further probe such mechanisms of enhanced permeation.

\section{Conclusion}

In the present study, CARS microscopy was used for the first time to image live intestinal epithelial cell cultures on membrane inserts that are used for intestinal drug permeation studies during pharmaceutical development. The suitability of various commonly used cell culture insert materials and imaging media for CARS imaging of live cells was evaluated. No differences in CARS spectra between different media solutions were observed, with HBSS- 


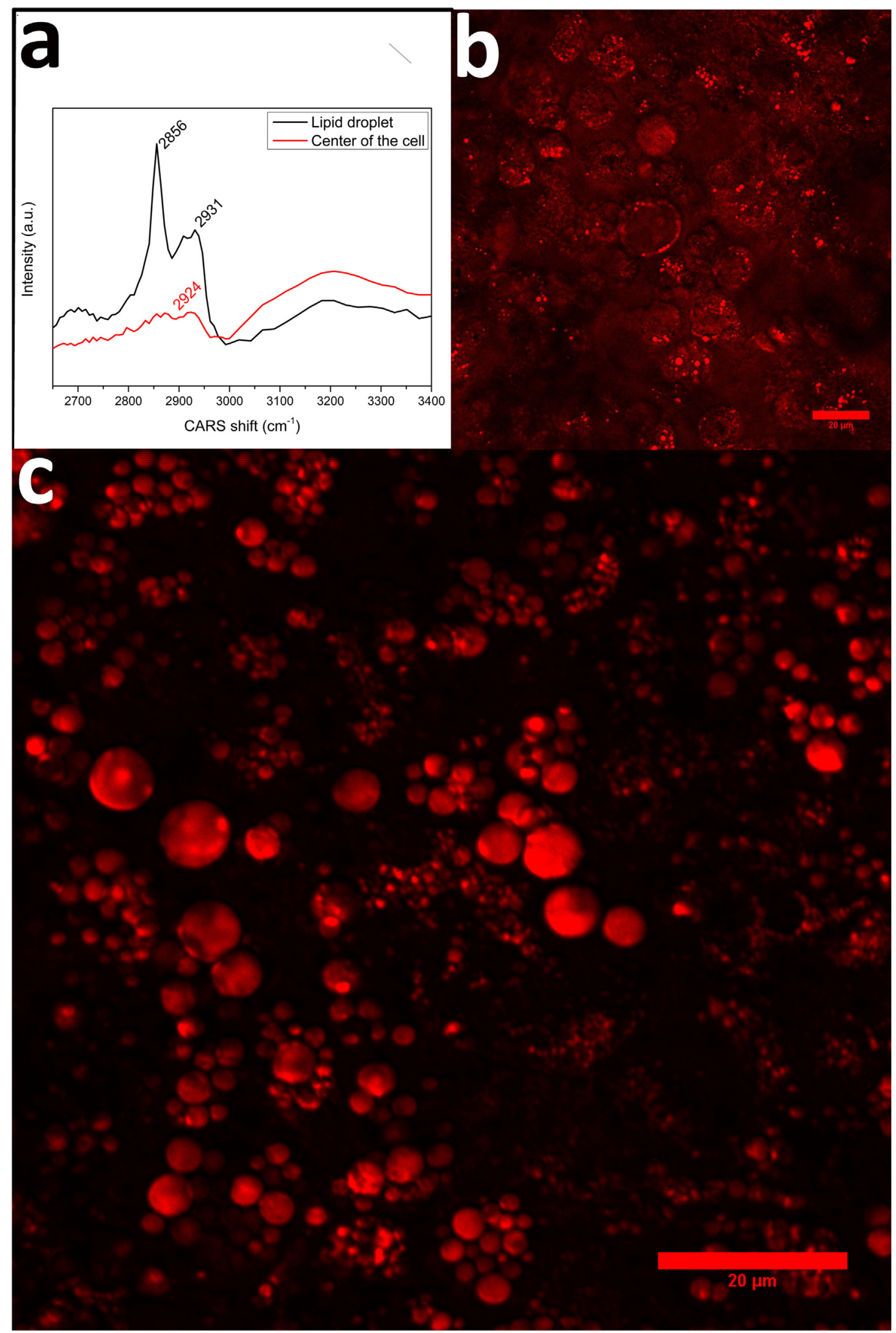

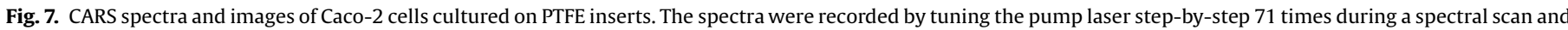

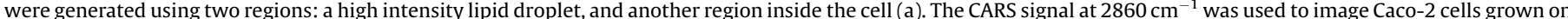

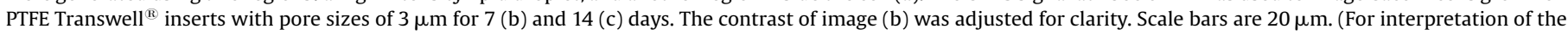
references to colour in this figure legend, the reader is referred to the web version of this article.) 
HEPES, PBS, and DMEM all able to be used as cell culture media while imaging. PTFE was the most robust membrane insert material for CARS imaging. In addition, the image quality was substantially better when the cells were imaged on PTFE inserts rather than the PE and PC inserts. When imaging live Caco- 2 cell cultures, it was found that the number and size of the lipid droplets increased substantially over a 21 day culturing period, which is important in the context of drug permeation studies.

This study represents the first application of a suitable labelfree imaging platform for live intestinal epithelial cell cultures in vitro. The CARS microscopy setup allows efficient, rapid and nondestructive in situ imaging in pharmaceutically relevent permeation testing conditions. As such a single culture may be used for both imaging and permeation studies. In addition to monitoring changes in single cultures during the culturing period as was done in this study, the platform may also be used to investigate cultureto-culture, batch-to-batch and lab-to-lab variability. Furthermore, the lipid content of Caco- 2 cells could be compared to intestinal epithelium tissue using CARS microscopy.

In future studies, cell models can be expanded to include other epithelial cell cultures and those that consist of a mixture of different cell types. Also, the interaction of drug nanoparticles with cells, which may influence drug absorption, could be imaged in vitro and in vivo. It is expected that the advantages and the increasing availability of CARS imaging will lead to its widespread use in (bio)pharmaceutical research.

\section{Acknowledgments}

JS, ES and CS acknowledge the University of Helsinki for a threeyear research project grant (2014-2016) and the Academy of Finland (grant no. 289398). HS acknowledges financial support from the Academy of Finland (grant nos. 252215 and 281300), the University of Helsinki, Biocentrum Helsinki, and the European Research Council under the European Union's Seventh Framework Programme (FP/2007-2013) (grant no. 310892).

\section{Appendix A. Supplementary data}

Supplementary data associated with this article can be found, in
online version, at http://dx.doi.org/10.1016/j. ijpharm.2017.03.015.

\section{References}

Accioly, M.T., Pacheco, P., Maya-Monteiro, C.M., Carrossini, N., Robbs, B.K., Oliveira, S S., Kaufmann, C., Morgado-Diaz, J.A., Bozza, P.T., Viola, J.P.B., 2008. Lipid bodies are reservoirs of cyclooxygenase-2 and sites of prostaglandin-E2 synthesis in colon cancer cells. Cancer Res. 68, 1732-1740. doi:http://dx.doi.org/10.1158/ 0008-5472.CAN-07-1999.

Artursson, P., Palm, K., Luthman, K., 2001. Caco-2 monolayers in experimental and theoretical predictions of drug transport. Adv. Drug Deliv. Rev. 46, 27-43.

Artursson, P.E.R., 1990. Epithelial transport of drugs in cell culture. I: a model for studying the passive diffusion of drugs over intestinal. J. Pharm. Sci. 79, 476482.

Bonn, M., Müller, M., Rinia, H. a., Burger, K.N.J., 2009. Imaging of chemical and physical state of individual cellular lipid droplets using multiplex CARS microscopy. J. Raman Spectrosc. 40, 763-769. doi:http://dx.doi.org/10.1002/ jrs.2253.

Chai, G.-H., Xu, Y., Chen, S.-Q., Cheng, B., Hu, F.-Q., You, J., Du, Y.-Z., Yuan, H., 2016 Transport mechanisms of solid lipid nanoparticles across Caco-2 cell monolayers and their related cytotoxicology. ACS Appl. Mater. Interfaces 8 , 5929-5940. doi:http://dx.doi.org/10.1021/acsami.6b00821.

Cheng, J.-X., Jia, Y.K., Zheng, G., Xie, X.S., 2002. Laser-scanning coherent anti-Stokes Raman scattering microscopy and applications to cell biology. Biophys. J. 83 502-509. doi:http://dx.doi.org/10.1016/S0006-3495(02)75186-2.

Donna, A.V., 2008. Variability in Caco-2 and MDCK cell-based intestinal permeability assays. J. Pharm. Sci. 97, 712-725. doi:http://dx.doi.org/10.1002/ jps.

Ducharme, N.A., Bickel, P.E., 2008. Lipid droplets in lipogenesis and lipolysis. Endocrinology 149, 942-949. doi:http://dx.doi.org/10.1210/en.2007-1713.
El-Mashtoly, S.F., Niedieker, D., Petersen, D., Krauss, S.D., Freier, E., Maghnouj, A., Mosig, A., Hahn, S., Kötting, C., Gerwert, K., 2014. Automated identification of subcellular organelles by coherent anti-stokes Raman scattering. Biophys. J. 106, 1910-1920. doi:http://dx.doi.org/10.1016/j.bpj.2014.03.025.

Evans, C.L., Xie, X.S., 2008. Coherent anti-stokes Raman scattering microscopy: chemical imaging for biology and medicine. Annu. Rev. Anal. Chem. (Palo Alto. Calif) 1, 883-909. doi:http://dx.doi.org/10.1146/annurev. anchem.1.031207.112754.

Evans, C.L., Potma, E.O., Puoris'haag, M., Côté, D., Lin, C.P., Xie, X.S., 2005. Chemical imaging of tissue in vivo with video-rate coherent anti-Stokes Raman scattering microscopy. Proc. Natl. Acad. Sci. U. S. A. 102, 16807-16812. doi:http://dx.doi. org/10.1073/pnas.0508282102.

Fu, Y., Wang, H., Shi, R., Cheng, J., 2006. Characterization of photodamage in coherent anti-Stokes Raman scattering microscopy. Opt. Express 14, 3942-3951.

Galli, R., Uckermann, O., Andresen, E.F., Geiger, K.D., Koch, E., Schackert, G., Steiner, G., Kirsch, M., 2014. Intrinsic indicator of photodamage during label-free multiphoton microscopy of cells and tissues. PLoS One 9, e110295. doi:http://dx. doi.org/10.1371/journal.pone.0110295.

Garrett, N.L., Lalatsa, a., Begley, D., Mihoreanu, L., Uchegbu, I.F., Schätzlein, a. G., Moger, J., 2012. Label-free imaging of polymeric nanomedicines using coherent anti-stokes Raman scattering microscopy. J. Raman Spectrosc. 43, 681-688. doi: http://dx.doi.org/10.1002/jrs.3170.

Hellerer, T., Axäng, C., Brackmann, C., Hillertz, P., Pilon, M., Enejder, A., 2007. Monitoring of lipid storage in Caenorhabditis elegans using coherent antiStokes Raman scattering (CARS) microscopy. Proc. Natl. Acad. Sci. 104, 1465814663. doi:http://dx.doi.org/10.1073/pnas.0703594104.

Hopt, a, Neher, E., 2001. Highly nonlinear photodamage in two-photon fluorescence microscopy. Biophys. J. 80, 2029-2036. doi:http://dx.doi.org/10.1016/S00063495(01)76173-5.

Hubatsch, I., Ragnarsson, E.G.E., Artursson, P., 2007. Determination of drug permeability and prediction of drug absorption in Caco-2 monolayers. Nat. Protoc. 2, 2111-2119. doi:http://dx.doi.org/10.1038/nprot.2007.303.

Jüngst, C., Klein, M., Zumbusch, A., 2013. Long-term live cell microscopy studies of lipid droplet fusion dynamics in adipocytes. J. Lipid Res. 54, 3419-3429. doi: http://dx.doi.org/10.1194/jlr.M042515.

Li, P., Nielsen, H.M., Müllertz, A., 2016. Impact of lipid-based drug delivery systems on the transport and uptake of insulin across caco-2 cell monolayers. J. Pharm. Sci. 105, 2743-2751. doi:http://dx.doi.org/10.1016/j.xphs.2016.01.006.

Lin, C.-Y., Suhalim, J.L., Nien, C.L., Miljković, M.D., Diem, M., Jester, J.V., Potma, E.O., 2011. Picosecond spectral coherent anti-Stokes Raman scattering imaging with principal component analysis of meibomian glands. J. Biomed. Opt. 16, 21104. doi:http://dx.doi.org/10.1117/1.3533716.

Magidson, V., Khodjakov, A., 2013. Circumventing photodamage in live-cell microscopy. Methods Cell Biol. 114, 545-560. doi:http://dx.doi.org/10.1016/ B978-0-12-407761-4 (00023-3. Circumventing).

Martin, S., Parton, R.G., 2006. Lipid droplets: a unified view of a dynamic organelle. Nat. Rev. Mol. Cell Biol. 7, 373-378.

Murphy, D., 2001. The biogenesis and functions of lipid bodies in animals, plants and microorganisms. Prog. Lipid Res. 40, 325-438. doi:http://dx.doi.org/10.1016/ S0163-7827(01)00013-3.

Nan, X., Potma, E.O., Xie, X.S., 2006. Nonperturbative chemical imaging of organelle transport in living cells with coherent anti-stokes Raman scattering microscopy. Biophys. J. 91, 728-735. doi:http://dx.doi.org/10.1529/biophysj.105.074534.

Neves, A.R., Queiroz, J.F., Costa Lima, S.A., Figueiredo, F., Fernandes, R., Reis, S., 2016. Cellular uptake and transcytosis of lipid-based nanoparticles across the intestinal barrier: relevance for oral drug delivery. J. Colloid Interface Sci. 463, 258-265. doi:http://dx.doi.org/10.1016/j.jcis.2015.10.057.

Panicker, C.Y., Varghese, H.T., Philip, D., Nogueira, H.I.S., 2006. FT-IR, FT-Raman and SERS spectra of pyridine-3-sulfonic acid. Spectrochim. Acta. A. Mol. Biomol. Spectrosc. 64, 744-747. doi:http://dx.doi.org/10.1016/j.saa.2005.06.048.

Paul, A., Chang, B.H.-J., Li, L., Yechoor, V.K., Chan, L., 2008. Deficiency of adipose differentiation-related protein impairs foam cell formation and protects against atherosclerosis. Circ. Res. 102, 1492-1501. doi:http://dx.doi.org/10.1161/ CIRCRESAHA.107.168070.

Pol, A., Gross, S.P., Parton, R.G., 2014. Biogenesis of the multifunctional lipid droplet: lipids, proteins, and sites. J. Cell Biol. 204, 635-646. doi:http://dx.doi.org/ $10.1083 /$ jcb.201311051.

Salo, V.T., Belevich, I., Li, S., Karhinen, L., Vihinen, H., Vigouroux, C., Magré, J., Thiele, C., Hölttä-Vuori, M., Jokitalo, E., Ikonen, E., 2016. Seipin regulates ER-lipid droplet contacts and cargo delivery. EMBO J. e201695170. doi:http://dx.doi.org/ 10.15252/embj.201695170.

Scalfi-Happ, C., Udart, M., Hauser, C., Rück, A., 2011. Investigation of lipid bodies in a colon carcinoma cell line by confocal Raman microscopy. Med. Laser Appl. 26, 152-157. doi:http://dx.doi.org/10.1016/j.mla.2011.08.002.

Shah, P., Jogani, V., Bagchi, T., Misra, A., 2006. Role of Caco-2 cell monolayers in prediction of intestinal drug absorption. Biotechnol. Prog. 22, 186-198. doi: http://dx.doi.org/10.1021/bp050208u.

Tauchi-Sato, K., Ozeki, S., Houjou, T., Taguchi, R., Fujimoto, T., 2002. The surface of lipid droplets is a phospholipid monolayer with a unique Fatty Acid composition. J. Biol. Chem. 277, 44507-44512. doi:http://dx.doi.org/10.1074/jbc. M207712200.

Thiam, A.R., Farese, R.V., Walther, T.C., 2013. The biophysics and cell biology of lipid droplets. Nat. Rev. Mol. Cell Biol. 14, 775-786. doi:http://dx.doi.org/10.1038/ nrm3699.

van Meer, G., 2001. Caveolin, cholesterol, and lipid droplets? J. Cell Biol. 152, 29-34. doi:http://dx.doi.org/10.1083/jcb.152.5.F29. 
Vogel, A., Noack, J., Hüttman, G., Paltauf, G., 2005. Mechanisms of femtosecond laser nanosurgery of cells and tissues. Appl. Phys. B 81, 1015-1047. doi:http://dx.doi. org/10.1007/s00340-005-2036-6.

Volkmer, A., Cheng, J.-X., Sunney Xie, X., 2001. Vibrational imaging with high sensitivity via epidetected coherent anti-Stokes raman scattering microscopy. Phys. Rev. Lett. 87, 23901. doi:http://dx.doi.org/10.1103/PhysRevLett.87.023901.

Wang, Z., Gui, C., Zhao, E., Wang, J., Li, X., Qin, A., Zhao, Z., Yu, Z., Tang, B.Z., 2016. Specific fluorescence probes for lipid droplets based on simple AIEgens. ACS
Appl. Mater. Interfaces 8, 10193-10200. doi:http://dx.doi.org/10.1021/ acsami.6b01282.

Winterhalder, M.J., Zumbusch, A., 2015. Beyond the borders - biomedical applications of non-linear Raman microscopy. Adv. Drug Deliv. Rev. 89,135-144. doi:http://dx.doi.org/10.1016/j.addr.2015.04.024.

Wong, C.S.Y., Robinson, I., Ochsenk, M.A., Arlt, J., Hossack, W.J., Crain, J., 2011. Changes to lipid droplet configuration in MCMV-infected fibroblasts: live cell imaging with simultaneous CARS and two-photon fluorescence microscopy. Biomed. Opt. Express 2, 2504-2516. 